\title{
The Vienna Cancer Stem Cell Club (VCSCC): First 10 Years and Future Perspectives
}

\author{
Peter Valent ${ }^{1,2}$, Thomas Grunt ${ }^{1,3}$, Heidrun Karlic ${ }^{1,4}$, Harald Herrmann ${ }^{1,5}$, Brigitte Marian ${ }^{1,6}$, Axel Schulenburg ${ }^{1,7}$, \\ Edgar Selzer ${ }^{1,5}$, Stefan Wöhrer ${ }^{1,7}$, Hubert Pehamberger, ${ }^{1,8}$, Ulrich Jäger ${ }^{1,2}$ and Christoph C. Zielinski ${ }^{1,3}$ \\ 1. Ludwig Boltzmann Cluster Oncology, Medical University of Vienna, Vienna 1090, Austria \\ 2. Department of Medicine I, Division of Hematology \& Hemostaseology, Medical University of Vienna, Vienna 1090, Austria \\ 3. Department of Medicine I, Division of Clinical Oncology, Medical University of Vienna, Vienna 1090, Austria \\ 4. Department of Internal Medicine III, Division of Hematology/Oncology, Hanusch Hospital, Vienna 1140, Austria \\ 5. Department of Radiation Oncology, Medical University of Vienna, Vienna 1090, Austria \\ 6. Department of Medicine I, Institute of Cancer Research; Medical University of Vienna, Vienna 1090, Austria \\ 7. Department of Medicine I, Stem Cell Transplantation Unit, Medical University of Vienna, Vienna 1090, Austria \\ 8. Department of Dermatology, Medical University of Vienna, Vienna 1090, Austria
}

Received: January 05, 2013 / Accepted: April 09, 2013 / Published: June 30, 2013.

\begin{abstract}
In 2002, the Vienna Cancer Stem Cell Club (VCSCC) was inaugurated by a group of scientists at the Medical University of Vienna, with the primary goal to initiate and promote cancer stem cell (CSC) research in Austria and to exploit knowledge from this new discipline in translational approaches. During the first years following inauguration, the VCSCC-community was small and left without major funding. However, over time the consortium was able to launch several major project-lines, supported in part by the National Science Funds, a Genome Program, and the Ludwig-Boltzmann Society. Today, the VCSCC provides a robust intellectual platform for ongoing research in the field of translational oncology and CSC-research in Austria. In addition, the VCSCC is connected to several major CSC-networks and centers in Europe and in North America, and is a well-recognized group in the field. The VCSCC also organized a series of CSC Meetings and Conferences, and contributed essentially to a recently published classification of CSC. There is also hope that the VCSCC-consortium will further advance the field of CSC research in the future, and will create novel concepts, with the ultimate aim to improve anti-cancer therapy through elimination, suppression, or long-term control of cancer-initiating cells.
\end{abstract}

Key words: Stem cells, cancer stem cells, molecular targets, targeted drug therapies, curative anti-cancer approaches.

\section{Introduction and Historical Aspects}

During the past 15 years, cancer stem cells (CSC) have been studied extensively in basic science and translational oncology [1-7]. As per definition, CSC but not their more mature progeny (daughter cells), are capable of propagating the neoplasm (cancer) in the natural host for unlimited time periods [1-3]. This definition implies that CSC have self-renewal and long-term proliferative capacity as well as a certain

Corresponding author: Peter Valent, M.D., professor, research fields: hematology and cancer stem cells. E-mail: peter.valent@meduniwien.ac.at. ability to undergo asymmetrical cell division and subsequent differentiation to form the mass of "more mature" elements of the tumor. Initially, the "CSC hypothesis" has been established in certain leukemia models, where the cellular hierarchy and CSC-dependence of the clone are obvious features $[1-5,8-12]$. Notably, CSC, referred to as leukemia-propagating or leukemic stem cells (LSC) in leukemic patients, have been identified and partly characterized in acute myeloid leukemia (AML) and Philadelphia chromosome-positive $\left(\mathrm{Ph}^{+}\right)$chronic myeloid leukemia (CML) [8-12]. In the chronic phase 
of CML, the clonal hierarchy is associated with a substantial differentiation- and maturation capacity of leukemic cells, which is a pathognomonic feature in CML. More recently, the CSC hypothesis has also been tested successfully in various models of solid tumors, malignant melanoma, lymphoma, and multiple myeloma [13-20]. The stem cell hierarchy is also demonstrable in these malignancies. However, in advanced solid tumors and melanomas, but also in the terminal stage of leukemias, the cellular hierarchy is "flat" compared to "earlier" phases of the disease. In addition, neoplastic stem cells are heterogeneous populations of cells in various tumors, and stemness may sometimes even be a reversible feature and not hierarchically organized in all subclones of a given neoplasm [21-27].

Clinically important issues in CSC research are the identification of CSC-related markers and oncogenic pathways, the identification of molecular targets in these cells, and the CSC-eradicating potential of various targeted drugs and drug combinations (Table 1) [28-32]. However, although diverse CSC markers, targets, and target pathways have been identified, several questions remain, such as the origin and evolution of CSC, mechanisms underlying resistance of CSC against conventional drugs or/and targeted drugs, and the molecular mechanisms that may contribute to molecular interactions between
CSC and microenvironmental (stromal) cells in the so-called "stem cell niche" [33-37].

An important aspect is that during disease evolution, CSC proliferation is gradually shifting towards symmetrical cell divisions. In addition, more and more molecular lesions (aberrations), epigenetic changes and posttranslational modifications are acquired by CSC. As a result, CSC in an overt tumor is quite heterogeneous populations of cells, with varying combinations of molecular lesions and oncogenic pathways, resulting in a variable tumorigenic potential of CSC-subpopulations [31, 38-43]. Subclone formation is based on genomic instability and plasticity of CSC, patient-related factors (such as the immune system) and the increased rate of symmetrical cell divisions that lead to a continuous expansion and diversification of the CSC pool [38-43]. Subclone formation is also considered to contribute essentially to acquired resistance against diverse types of drugs (multidrug resistance) [38-43]. Notably, CSC often remain unresponsive to many different drugs because of "intrinsic" (natural) stem cell resistance combined with multiple (additional) mechanisms of "acquired (subclone-specific) resistance" [31, 40]. Finally, several different interactions between CSC and the so-called stem cell niche, but also between CSC and the immune system, may contribute to drug resistance in CSC [35-37].

Table 1 Major issues in cancer stem cell research.

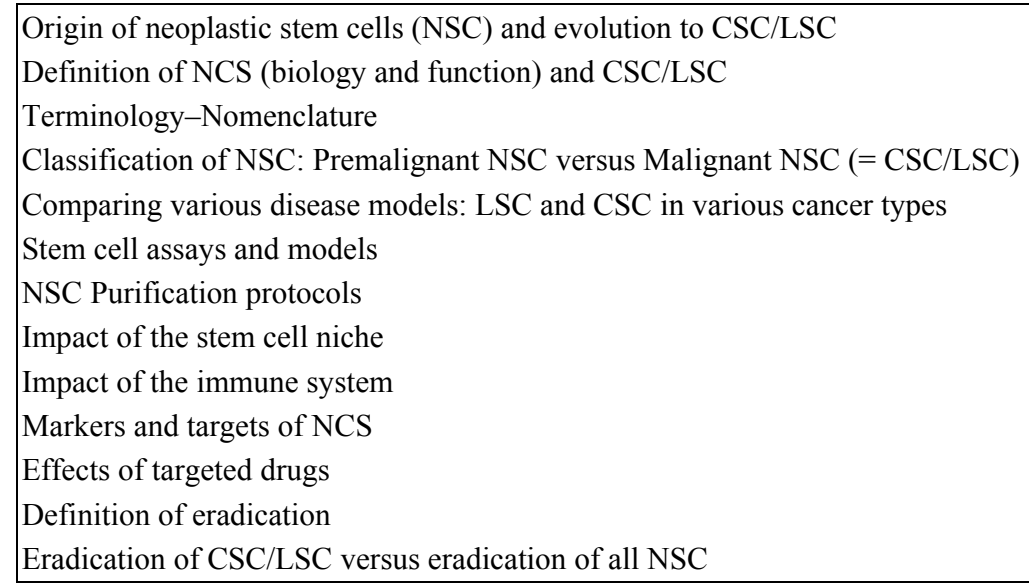


Whereas research on CSC has developed rapidly in various countries in the past decade, this field of science was not expanding in Austria for quite a long time. However, between 2002 and 2005, first attempts were made to establish robust CSC assays in various disease models in several institutions in Austria. One essential step forward was the establishment of robust human tumor cell xenotransplantation models using highly immunodeficient mice.

In the year 2002, a local group of experts working in the field of cancer research at the Medical University of Vienna, started to meet regularly (once per week Monday afternoon) to discuss theoretical and practical (translational) aspects of CSC research and various ongoing projects. After some months, the group decided to implement this meeting-platform by introducing the Vienna Cancer Stem Cell Club (VCSCC). The VCSCC was inaugurated in November 2002.

During the past 10 years, the VCSCC expanded substantially, and started a series of projects in CSC research in Vienna. After some years, members of the VCSCC were able to organize funding for their research on CSC and to publish major results on the biology and function of CSC. In addition, members of the VCSCC organized a series of meetings and conferences between 2002 and 2012. The aims, achievements, meetings and future perspectives of the VCSCC are summarized in this article.

\section{Major Aims of the VCSCC}

A general strategic aim of the VCSCC is to provide a solid multi-disciplinary platform for interactive discussions and cooperative research on CSC in Vienna. A related specific aim is to assist in the initiation of specific cooperations in the field of CSC research, and to intensify and assist ongoing collaborations with national and international partners working on CSC. To reach these goals, the VCSCC has established a weekly jour fix meeting to discuss novel developments in the field and new results in ongoing projects and cooperations. In addition, the VCSCC has organized smaller joint meetings between research groups in order to initiate new, and to discuss ongoing, collaborative CSC projects in Vienna. Another important aim of the VCSCC is to attract sponsors and to organize budget for CSC research at the Medical University of Vienna. In these collaborative projects, drug targets expressed in CSC and the effects of various targeted drugs acting on (and eliminating) $\mathrm{CSC}$, have been examined. An important aim of the VCSCC is to educate young scientists interested in CSC research, to promote junior group leaders in the field, and to motivate clinical colleagues to translate CSC concepts and results arising from ongoing $\mathrm{CSC}$ research into interventional clinical trials (translation). With regard to clinical trials and translational projects, the VCSCC exploits a strong local network of clinical working groups and the many interactions between the Medical University of Vienna and other clinical study groups as well as diverse industrial partners. Moreover, the VCSCC cooperates with the Comprehensive Cancer Center (CCC) of the Medical University of Vienna. Finally, the VCSCC is connected to the Ph.D. program of the Medical University of Vienna and with several international working groups and EU projects, including an EU training network program focusing on CSC research.

\section{Major Topics and Project Lines}

At the time of initiation in 2002, three major project lines were launched: one focusing on myeloid neoplasms, one on mast cell disorders, and one on solid tumors. In the year 2008, two additional project lines were initiated, namely one dealing with lymphoproliferative neoplasms and one focusing on melanomas. Each project line includes several different research groups and supports a number of defined cooperative research efforts. Depending on the expertise of the group, developments in the field, and available tools and funding, the VCSCC decided 
to focus on certain disease-models in each cancer group. Among myeloid neoplasms, the VCSCC has a focus in acute myeloid leukemia (AML), myelodysplastic syndromes (MDS) and chronic myeloid leukemia (CML). Within mast cell disorders, the VCSCC works on the characterization of neoplastic (stem) cells in mast cell leukemia (MCL). Within the field of solid tumors, the VCSCC focuses primarily on colon adenomas and colon carcinomas and on certain gynecological tumors. Among lymphoproliferative neoplasms, chronic lymphocytic leukemia (CLL) and acute lymphoblastic leukemia (ALL) were selected as major disease models. In addition, the VCSCC focused on advanced skin melanomas. In each project line, the VCSCC managed to organize constant access to primary tumor cell material and to establish robust xenotransplantation models through collaborations with various scientific partners and groups at the Medical University of Vienna, the Veterinary University of Vienna and other major institutions in Vienna.

\section{Structure and Regular Meetings}

The VCSCC is a non-profit cooperative platform of scientists without any legal status. Members of the VCSCC are not obliged to any duties or responsibilities, and all input and contribution to the VCSCC is provided on a voluntary basis. The VCSCC meets once a week every Monday at 5 p.m. at the Medical University of Vienna. These meetings represent a valuable platform for scientific discussions and collaborations. Novel projects are introduced and ongoing projects are discussed on a regular basis: projects on myeloid neoplasms in the first week (first Monday) in each month, lymphoid neoplasms in the second, solid tumors in the third, and melanomas and mast cell tumors in the fourth week of each month. The VCSCC also hosts a weekly journal club and a seminar series of the Ludwig Boltzmann Cluster Oncology (LB-CO) of Vienna. Finally, the VCSCC has organized a number of smaller and larger scientific meetings, including a series of working conferences and international top meetings on CSC in the past 10 years (Table 2 and text below).

\section{Established Cooperations and Networking}

The VCSCC has established numerous cooperations with local, national and international groups working in the field of CSC research, various academic organizations and societies in the field of oncology and hematology. One good example is the European Hematology Association (EHA). The VCSCC has several active collaborations within the EHA and P.V. is co-chairing a scientific working group ( $\mathrm{SWG}$ ) on $\mathrm{CSC}$ in this rapidly growing Medical Society. The VCSCC has also established several important collaborations with suitable industrial partners interested in CSC research. Based on the mutual interest of both partners, many of these collaborations are rapidly expanding or have been intensified in the past few years. Whereas the academic partners are able to test new innovative drugs against CSC, the industrial partners may learn whether their drugs may indeed have a curative (CSC-eliminating) potential

Table 2 Meetings organized, co-organized or initiated by the VCSCC.

First Vienna Cancer Stem Cell Meeting 2004
Second Vienna Cancer Stem Cell Meeting 2008
Ludwig Boltzmann Cluster Oncology (LB-CO) Meeting 2010
ESH-EHA Scientific Workshop on Cancer Stem Cells 2011
Year 2011 Working Conference on Cancer Stem Cells
EHA Cancer Stem Cell SWG Meetings 2010, 2011, 2012
10 Year Jubilee Meeting of the VCSCC-Vienna 2012
ESH-EHA Scientific Workshop on Cancer Stem Cells 2013
VCSCC, Vienna Cancer Stem Cell Club; ESH, European School of Hematolgy; EHA, European Hematology Association; SWG,
Scientific Working Group.




\section{Major Events and Conferences}

The first Cancer Stem Cell Meeting in Vienna was organized by the VCSCC in 2004 (Table 2). Several pioneers in the field of CSC research, including Connie Eaves and Michael Clarke, participated. The meeting was well organized and served as a starting-point of CSC research for several groups in Austria. A second Cancer Stem Cell Meeting was organized by the VCSCC in the year 2008 (Table 2). Again, numerous authorities in the field participated and the event was a great success. In this meeting, the Vienna VCSCC consortium was presented to the scientific community for the first time. It turned out that similar networks also exist or are just being established in several other countries in Europe, such as in France (Paris), but also in North America. In 2011, the VCSCC organized the Year 2011 Working Conference on CSC (Table 2). In this meeting, a panel of authorities in the field discussed various aspects of CSC research, including terminologies, the biology and heterogeneity of CSC, as well as limitations of concepts and assays [40]. Furthermore, a new classification of CSC was proposed by the faculty. Based on this proposal, neoplastic stem cells (the umbrella term) can be divided into premalignant (in leukemias: pre-leukemic) neoplastic stem cells and malignant (leukemic) neoplastic stem cells [40]. Premalignant (pre-leukemic) neoplastic stem cells can produce a premalignant neoplastic condition (e.g., a myelodysplastic syndrome or colona adenoma) but not a fully transformed malignancy (e.g., acute leukemia or colon carcinoma). However, over time, premalignant neoplastic stem cells may transform into fully malignant neoplastic stem cells (= CSC), depending on additional lesions and hits and epigenetic modifications [40-44]. Thus, stem cell evolution is a multi-faceted and multi-step process that takes years or even decades, and is propagated in one, more, or many different (separately developing) subclones. This new concept and classification of neoplastic stem cells may explain a number of different clinical phenomena, such as the extensive plasticity and heterogeneity of primary and secondary cancer lesions, lineage switches of cancer cells, or the different biology and resistance of relapsing disease [40, 43, 45-47]. The faculty delivered consensus statements and the proposed classification in 2012 [40]. In the meantime, this new concept has received broader acceptance in the field [41-43]. In October 2012, the VCSCC celebrated its 10 Year Jubilee in form of a Scientific Workshop in the "Billroth Haus" in Vienna (Table 2).

\section{Other Scientific Highlights}

During the past 10 years, the VCSCC has accumulated a number of scientific results. Major achievements include the development of theoretical concepts around CSC, the phenotypic characterization of CSC and LSC in various disease models, the identification of specific stem cell markers, the identification of molecular targets in neoplastic stem cells and the evaluation of drug responses. Putative LSC were identified in $\mathrm{Ph}^{+}$CML, CLL, MDS, AML, ALL, colon cancer and malignant melanoma [48-54]. As assessed by gene chip and antibody-screen analyses, several new stem cell antigens, such as roundabout-4 (ROBO4), the IL-2 receptor alpha chain (CD25), dipeptidyl-peptidase IV (DPPIV $=\mathrm{CD} 26)$, Siglec-3 (CD33) or Campath-1 (CD52), were identified in these studies [48-54]. An interesting observation was that in all overt leukemias, including even CLL, NSC reside within the $\mathrm{CD} 34^{+}$portion of the malignant clone. Another interesting observation was that several markers identified on leukemic stem cells are also detectable on stem cell populations in pre-leukemic conditions and even also in non-hematologic (solid) tumors. Several of the newly identified surface markers on LSC/CSC may also serve as molecular targets (Table 3). Examples are CD25, CD26, Siglec-3 (CD33) and the Campath-1 (CD52) antigen. Target validation using shRNA or 
Table 3 Some major surface targets identified on LSC in AML or/and CML.

\begin{tabular}{lcl}
\hline Surface target & CD & Targeted drug (s) \\
\hline IL-2RA & 25 & Denileukin diftitox \\
DPPIV & 26 & Gliptins (various on the market) \\
SIGLEC 3 & 33 & Gemtuzumab ozogamicin \\
IAP & 47 & Targeted antibodies \\
CAMPATH 1 & 52 & Alemtuzumab \\
KIT & 117 & Imatinib and other TKI \\
IL-3RA & 123 & Various targeted antibodies \\
CLL-1 & n.c. & Targeted antibodies \\
IL-1RAP & n.c. & Targeted antibodies \\
ROBO4 & n.c. & Not available \\
\hline CSC,
\end{tabular}

CSC, cancer stem cells; LSC, leukemic stem cells; AML, acute myeloid leukemia; CML, chronic myeloid leukemia; IL-2RA, interleukin-2 receptor alpha chain; DPPIV, dipeptidylpeptidase IV; IAP, integrin-associated protein; TKI, tyrosine kinase inhibitors; n.c., not yet clustered.

targeted drugs was performed in several instances, and in each case, targeting of LSC/CSC was followed by their elimination. Unfortunately, however, some of these antigens are also expressed on normal stem cells or normal immune cells relevant to immune surveillance. Therefore, these antibody-type drugs also produce side effects, such as prolonged cytopenias or immunodeficiency. As a result, some of these agents, although very effective as anti-leukemic agents, like the CD33-targeted drug gemtuzumab-ozogamicin (mylotarg) or the CD52-targeted drug alemtuzumab (mabcampath), have recently been removed from the market.

During the past few years, members of the VCSCC have started to explore the mechanisms underlying the interactions between cancer (leukemic) stem cells and the organ- and disease-specific microenvironment, the so-called stem cell niche. In these analyses, several new important interactions have been discovered. An example is CD26, also known as di-deptidylpeptidase IV, an enzyme that degrades SDF-1-alpha and thereby disrupts specific interactions between CSC and the stem cell niche [52]. In addition, the VCSSC has a strong interest in epigenetic aspects of myeloid neoplasms and their LSC [54-56]. Another important aim of the VCSCC is to explore mechanisms contributing to intrinsic and acquired resistance of CSC/LSC against various (targeted or conventional) anti-neoplastic drugs. One key of our understanding drug resistance is the new model of CSC/LSC subclone formation. This model predicts that unlike considered previously, many different types or even all of the CSC/LSC may be reached and even eliminated completely by a certain drug or drug combination. Still, however, this does not mean that all premalignant neoplastic stem cells (NSC) were killed. Notably, premalignant NSC exhibit a particular intrinsic resistance which may result from their dormancy. After an initially successful therapy, the residual premalignant NSC thus serve as a reservoir of stem cells and after a certain latency period, these cells may transform into fully malignant CSC/LSC and thus are responsible for a (late) relapse. Members of the VCSCC are running several projects in which the biology and the mechanisms underlying resistance of premalignant NSC are examined. In addition, VCSCC projects aim at identifying mechanisms and molecular events contributing to the transformation of premalignant NSC into fully malignant CSC/LSC. The consortium has recently established a special research program (Spezialforschungsbereich-SFB) in Vienna in order to explore such mechanisms in myeloproliferative neoplasms. Finally, the VCSSC has the aim to explore the genetic and epigenetic complexity and the signaling maschineries underlying cancer stem cell evolution in myeloid neoplasms and other malignancies. 


\section{Summary and Future Perspectives}

The VCSCC was established with the intention to initiate and expand CSC research in Vienna and Austria, with the hope to exploit arising concepts in translational oncology, and with the ultimate aim to improve anticancer therapy through elimination or complete control of tumor-initating cells. To address these aims, the VCSCC has launched several project lines and organized a series of meetings and conferences in the past 10 years. Visible progress has been made in several of these project-lines and there is now hope for the future that these concepts can be translated into clinical practice. The VCSCC will continue to support most important developments in the field of CSC, with a major focus on translational aspects in hematology and oncology.

\section{References}

[1] T. Reya, S.J. Morrison, M.F. Clarke, I.L. Weissman, Stem cells, cancer, and cancer stem cells, Nature 414 (2001) 105-111.

[2] R. Pardal, M.F. Clarke, S.J. Morrison, Applying the principles of stem-cell biology to cancer, Nat Rev Cancer 3 (2003) 895-902.

[3] K. Polyak, W.C. Hahn, Roots and stems: Stem cells in cancer, Nat Med 12 (2006) 296-300.

[4] M.L. Guzman, C.T. Jordan, Considerations for targeting malignant stem cells in leukemia, Cancer Control 11 (2004) 97-104.

[5] W.R. Sperr, A.W. Hauswirth, S. Florian, L. Ohler, K. Geissler, P. Valent, Human leukaemic stem cells: A novel target of therapy, Eur J Clin Invest 34 (S2) (2004) 31-40.

[6] M. Al-Hajj, M.S. Wicha, A. Benito-Hernandez, S.J. Morrison, M.F. Clarke, Prospective identification of tumorigenic breast cancer cells, Proc Natl Acad Sci (USA) 100 (2003) 3983-3988.

[7] A. Schulenburg, H. Ulrich-Pur, D. Thurnher, B. Erovic, S. Florian, W.R. Sperr, et al., Neoplastic stem cells: A novel therapeutic target in clinical oncology, Cancer 107 (2006) 2512-2520.

[8] T. Lapidot, C. Sirard, J. Vormoor, B. Murdoch, T. Hoang, J. Caceres-Cortes, et al., A cell initiating human acute myeloid leukaemia after transplantation into SCID mice, Nature 367 (1994) 645-648.

[9] D. Bonnet, J.E. Dick, Human acute myeloid leukemia is organized as a hierarchy that originates from a primitive hematopoietic cell, Nat Med 3 (1997) 730-737.
[10] K.J. Hope, L. Jin, J.E. Dick, Acute myeloid leukemia originates from a hierarchy of leukemic stem cell classes that differ in self-renewal capacity, Nat Immunol 5 (2004) 738-743.

[11] X. Jiang, K.M. Saw, A. Eaves, C. Eaves, Instability of BCR-ABL gene in primary and cultured chronic myeloid leukemia stem cells, J Natl Cancer Inst 99 (2007) 680-693.

[12] E. Kavalerchik, D. Goff, C.H. Jamieson, Chronic myeloid leukemia stem cells, J Clin Oncol 26 (2008) 2911-2915.

[13] L. Ricci-Vitiani, D.G. Lombardi, E. Pilozzi, M. Biffoni, M. Todaro, C. Peschle, et al., Identification and expansion of human colon-cancer-initiating cells, Nature 445 (2007) 111-115.

[14] C.A. O'Brien, A. Pollett, S. Gallinger, J.E. Dick, A human colon cancer cell capable of initiating tumour growth in immunodeficient mice, Nature 445 (2007) 106-110.

[15] S.K. Singh, I.D. Clarke, M. Terasaki, V.E. Bonn, C. Hawkins, J. Squire, et al., Identification of a cancer stem cell in human brain tumors, Cancer Res 63 (2003) 5821-5828

[16] M.E. Prince, R. Sivanandan, A. Kaczorowski, G.T. Wolf, M.J. Kaplan, P. Dalerba, et al., Identification of a subpopulation of cells with cancer stem cell properties in head and neck squamous cell carcinoma, Proc Natl Acad Sci (USA) 104 (2007) 973-978.

[17] T. Schatton, G.F. Murphy, N.Y. Frank, K. Yamaura, A.M. Waaga-Gasser, M. Gasser, et al., Identification of cells initiating human melanomas, Nature 451 (2008) 345-349.

[18] S.E. Zabierowski, M. Herlyn, Melanoma stem cells: The dark seed of melanoma, J Clin Oncol 26 (2008) 2890-2894.

[19] C.A. Huff, W. Matsui, Multiple myeloma cancer stem cells, J Clin Oncol 26 (2008) 2895-2900.

[20] Y. Kikushige, F. Ishikawa, T. Miyamoto, T. Shima, S. Urata, G. Yoshimoto, et al., Self-renewing hematopoietic stem cell is the primary target in pathogenesis of human chronic lymphocytic leukemia, Cancer Cell 20 (2011) 246-259.

[21] E. Quintana, M. Shackleton, M.S. Sabel, D.R. Fullen, T.M. Johnson, S.J. Morrison, Efficient tumour formation by single human melanoma cells, Nature 456 (2008) 593-598.

[22] C.J. Eaves, Cancer stem cells: Here, there, everywhere? Nature 456 (2008) 581-582.

[23] E. Quintana, M. Shackleton, H.R. Foster, D.R. Fullen, M.S. Sabel, T.M. Johnson, et al., Phenotypic heterogeneity among tumorigenic melanoma cells from patients that is reversible and not hierarchically organized, Cancer Cell 18 (2010) 510-523.

[24] D.C. Taussig, F. Miraki-Moud, F. Anjos-Afonso, D.J. 
Pearce, K. Allen, C. Ridler, et al., Anti-CD38 antibody-mediated clearance of human repopulating cells masks the heterogeneity of leukemia-initiating cells, Blood 112 (2008) 568-575.

[25] A. Roesch, M. Fukunaga-Kalabis, E.C. Schmidt, S.E. Zabierowski, P.A. Brafford, A. Vultur, et al., A temporarily distinct subpopulation of slow-cycling melanoma cells is required for continuous tumor growth, Cell 141 (2010) 583-594.

[26] D.C. Taussig, J. Vargaftig, F. Miraki-Moud, E. Griessinger, K. Sharrock, T. Luke, et al., Leukemia-initiating cells from some acute myeloid leukemia patients with mutated nucleophosmin reside in the CD34(') fraction, Blood 115 (2010) 1976-1984.

[27] N. Goardon, E. Marchi, A. Atzberger, L. Quek, A. Schuh, S. Soneji, et al., Coexistence of LMPP-like and GMP-like leukemia stem cells in acute myeloid leukemia, Cancer Cell 19 (2011) 138-152.

[28] M. Al-Hajj, M.W. Becker, M. Wicha, I. Weissman, M.F. Clarke, Therapeutic implications of cancer stem cells, Curr Opin Genet Dev 14 (2004) 43-47.

[29] A. Trumpp, O.D. Wiestler, Mechanisms of disease: Cancer stem cells-targeting the evil twin, Nat Clin Pract Oncol 5 (2008) 337-347.

[30] G.V. Helgason, G.A. Young, T.L. Holyoake, Targeting chronic myeloid leukemia stem cells, Curr Hematol Malig Rep 5 (2010) 81-87.

[31] P. Valent, Targeting of leukemia-initiating cells to develop curative drug therapies: Straightforward but nontrivial concept, Curr Cancer Drug Targets 11 (2011) 56-71.

[32] J.A. McCubrey, L.S. Steelman, S.L. Abrams, N. Misaghian, W.H. Chappell, J. Basecke, et al., Targeting the cancer initiating cell: the ultimate target for cancer therapy, Curr Pharm Des 18 (2012) 1784-1795.

[33] D.J. Barnes, J.V. Melo, Primitive, quiescent and difficult to kill: The role of non-proliferating stem cells in chronic myeloid leukemia, Cell Cycle 5 (2006) 2862-2866.

[34] X. Jiang, Y. Zhao, C. Smith, M. Gasparetto, A. Turhan, A. Eaves, et al., Chronic myeloid leukemia stem cells possess multiple unique features of resistance to BCR-ABL targeted therapies, Leukemia 21 (2007) 926-935.

[35] A. Singh, J. Settleman, EMT, cancer stem cells and drug resistance: An emerging axis of evil in the war on cancer, Oncogene 29 (2010) 4741-4751.

[36] R.R. Nair, J. Tolentino, L.A. Hazlehurst, The bone marrow microenvironment as a sanctuary for minimal residual disease in CML, Biochem Pharmacol 80 (2010) 602-612.

[37] M.Y. Konopleva, C.T. Jordan, Leukemia stem cells and microenvironment: Biology and therapeutic targeting, $\mathrm{J}$
Clin Oncol 29 (2011) 591-599.

[38] M. Greaves, Cancer stem cells: Back to Darwin?, Semin Cancer Biol 20 (2010) 65-70.

[39] E. Clappier, B. Gerby, F. Sigaux, M. Delord, F. Touzri, L. Hernandez, et al., Clonal selection in xenografted human $\mathrm{T}$ cell acute lymphoblastic leukemia recapitulates gain of malignancy at relapse, J Exp Med 208 (2011) 653-661.

[40] P. Valent, D. Bonnet, R. De Maria, T. Lapidot, M. Copland, J.V. Melo, et al., Cancer stem cell definitions and terminology: The devil is in the details, Nat Rev Cancer 12 (2012) 767-775.

[41] S.J. Horton, B.J. Huntly, Recent advances in acute myeloid leukemia stem cell biology, Haematologica 97 (2012) 966-974.

[42] M. Jan, T.M. Snyder, M.R. Corces-Zimmerman, P. Vyas, I.L. Weissman, S.R. Quake, et al., Clonal evolution of preleukemic hematopoietic stem cells precedes human acute myeloid leukemia, Sci Transl Med 4 (2012) 149ra118.

[43] P. Valent, D. Bonnet, S. Wöhrer, M. Andreeff, M. Copland, C. Chomienne, et al., Heterogeneity of neoplastic stem cells: Theoretical, functional and clinical implications, Cancer Res 73 (2013) 1037-1045.

[44] M. Widschwendter, H. Fiegl, D. Egle, E. Mueller-Holzner, G. Spizzo, C. Marth, et al., Epigenetic stem cell signature in cancer, Nat Genet 39 (2007) 157-158.

[45] L. Ding, M.J. Ellis, S. Li, D.E. Larson, K. Chen, J.W. Wallis, et al., Genome remodelling in a basal-like breast cancer metastasis and xenograft, Nature 464 (2010) 999-1005.

[46] M.J. Walter, D. Shen, L. Ding, J. Shao, D.C. Koboldt, K. Chen, et al., Clonal architecture of secondary acute myeloid leukemia, N Engl J Med 366 (2012) 1090-1098.

[47] L. Ding, T.J. Ley, D.E. Larson, C.A. Miller, D.C. Koboldt, J.S. Welch, et al., Clonal evolution in relapsed acute myeloid leukaemia revealed by whole-genome sequencing, Nature 481 (2012) 506-510.

[48] S. Florian, K. Sonneck, A.W. Hauswirth, M.T. Krauth, G.H. Schernthaner, W.R. Sperr, et al., Detection of molecular targets on the surface of $\mathrm{CD} 34^{+} / \mathrm{CD} 38^{-}$stem cells in various myeloid malignancies, Leuk Lymphoma 47 (2006) 207-222.

[49] A.W. Hauswirth, S. Florian, D. Printz, K. Sotlar, M.T. Krauth, G. Fritsch, et al., Expression of the target receptor $\mathrm{CD} 33$ in $\mathrm{CD} 34^{+} / \mathrm{CD} 38^{-} / \mathrm{CD} 123^{+}$AML stem cells, Eur J Clin Invest 37 (2007) 73-82.

[50] A. Schulenburg, P. Cech, I. Herbacek, M. Marian, F. Wrba, P. Valent, et al., CD44-positive colorectal adenoma cells express the potential stem cell markers musashi antigen (msi1) and ephrin B2 receptor (EphB2), J Pathol 213 (2007) 152-160. 
[51] P. Valent, S. Cerny-Reiterer, H. Herrmann, I. Mirkina, T.I. George, K. Sotlar, et al., Phenotypic heterogeneity, novel diagnostic markers, and target expression profiles in normal and neoplastic human mast cells, Best Pract Res Clin Haematol 23 (2010) 369-378.

[52] H. Herrmann, I. Sadovnik, S. Cerny-Reiterer, K. Blatt, V. Ghanim, S. Herndlhofer, et al., The Leukemic Stem Cell (LSC) in $\mathrm{Ph}^{+} \mathrm{CML}$ Is a $\mathrm{CD} 34^{+} / \mathrm{CD} 38^{-} / \mathrm{Lin}^{-}$Cell That Co-Expresses Dipeptidylpeptidase IV (CD26) and Disrupts LSC-Niche Interactions by Degrading the CXCR4 Ligand SDF-1 $\alpha$, Blood 118 (2011) 961.

[53] H. Herrmann, S. Cerny-Reiterer, K.V. Gleixner, K. Blatt, S. Herndlhofer, W. Rabitsch, et al., CD $34\left(^{+}\right) / \mathrm{CD} 38\left(^{\circ}\right)$ stem cells in chronic myeloid leukemia express Siglec-3 (CD33) and are responsive to the CD33-targeting drug gemtuzumab/ozogamicin, Haematologica 97 (2012) 219-226.

[54] T. Grunt, A. Hebar, S. Laffer, R. Wagner, B. Peter, H. Herrmann, et al., Prominin-1/CD133 is associated with CD26-expression and with a growth advantage in HCT116 colon cancer cells, but is not responsible for proliferation, survival, or drug resistance, manuscript submitted.

[55] I. Mirkina, C. Krepler, M. Mikula, D. Mechtcheriakova, S. Strommer, E. Hadzijusufovic, et al., Phenotyping of human melanoma cells reveals a unique composition of receptor targets and a stem cell-like fraction co-expressing ErbB4, EPO-R and NGF-R, manuscript submitted.

[56] H. Karlic, H. Herrmann, A. Schulenburg, T.W. Grunt, S. Laffer, I. Mirkina, et al., Tumor stem cell research-basis and challenge for diagnosis and therapy, Wien Klin Wochenschr 122 (2010) 423-436.

[57] J. Zuber, J. Shi, E. Wang, A.R. Rappaport, H. Herrmann, E.A. Sison, et al., RNAi screen identifies $\operatorname{Brd} 4$ as a therapeutic target in acute myeloid leukaemia, Nature 478 (2011) 524-528.

[58] H. Herrmann, K. Blatt, J. Shi, K.V. Gleixner, S. Cerny-Reiterer, L. Müllauer, et al., Small-molecule inhibition of BRD4 as a new potent approach to eliminate leukemic stem- and progenitor cells in acute myeloid leukemia AML, Oncotarget 3 (2012) 1588-1599. 